\title{
The Distribution of the Maximum of a Gaussian Process: Rice Method Revisited.
}

\author{
Jean-Marc Azaïs, azais@cict.fr \\ Mario Wschebor * † wscheb@fcien.edu.uy
}

December 21, 2000

\begin{abstract}
This paper deals with the problem of obtaining methods to compute the distribution of the maximum of a one-parameter stochastic process on a fixed interval, mainly in the Gaussian case. The main point is the relationship between the values of the maximum and crossings of the paths, via the socalled Rice's formulae for the factorial moments of crossings.

We prove that for some general classes of Gaussian process the so-called "Rice series" is convergent and can be used for to compute the distribution of the maximum. It turns out that the formulae are adapted to the numerical computation of this distribution and becomes more efficient than other numerical methods, namely simulation of the paths or standard bounds on the tails of the distribution.

We have included some relevant numerical examples to illustrate the power of the method.
\end{abstract}

Mathematics Subject Classification (1991): 60Gxx, 60E05, 60G15, $65 \mathrm{U} 05$. Key words: Extreme Values, Distribution of the Maximum, Crossings of a Level, Rice's Formulae.

Short Title: Distribution of the Maximum.

*Laboratoire de Statistique et Probabilités. UMR-CNRS C55830 Université Paul Sabatier. 118, route de Narbonne. 31062 Toulouse Cedex 4. France.

†Centro de Matemática. Facultad de Ciencias. Universidad de la República. Calle Igua 4225. 11400 Montevideo. Uruguay. 


\section{Introduction}

Let $X=\left\{X_{t}: t \in \mathbb{R}\right\}$ be a stochastic process with real values and continuous paths defined on a probability space $(\Omega, \Im, P)$ and $M_{T}:=\max \left\{X_{t}: t \in[0, T]\right\}$.

The computation of the distribution function of the random variable $M_{T}$

$$
F(T, u):=P\left(M_{T} \leq u\right), u \in \mathbb{R}
$$

by means of a closed formula based upon natural parameters of the process $X$ is known only for a very restricted number of stochastic processes (and trivial functions of them): the Brownian Motion $\left\{W_{t}: t \geq 0\right\}$; the Brownian Bridge, $B_{t}:=W_{t}-$ $t W_{1}(0 \leq t \leq 1) ; B_{t}-\int_{0}^{1} B_{s} d s$ (Darling, 1983); the Brownian Motion with a linear drift (Shepp, 1979); $\int_{0}^{t} W_{s} d s+y t$ (McKean, 1963, Goldman, 1971 Lachal, 1991); the stationary Gaussian processes with covariance equal to:

1. $r(t)=e^{-|t|}$ (Ornstein-Uhlenbeck process, DeLong, 1981),

2. $r(t)=(1-|t|)^{+}$, T a positive integer (Slepian process, Slepian 1961, Shepp, 1971),

3. $r(t)$ even, periodic with with period $2, r(t)=1-\alpha|t|$ for $0 \leq|t| \leq 1,0<\alpha \leq 2$, (Shepp and Slepian 1976),

4. $r(t)=1-|t| / 1-\beta \vee-\beta / 1-\beta,|t|<1-\beta / \beta, 0<\beta \leq 1 / 2, T=(1-\beta) / \beta$ (Cressie 1980),

5. $r(t)=\cos t$.

Given the interest in $F(T, u)$ for a large diversity of theoretical and technical purposes an extensive literature has been developed of which we give a sample of references pointing to various directions:

1. Obtaining inequalities for $F(T, u)$ : Slepian (1962); Landau \& Shepp (1970); Marcus \& Shepp (1972); Fernique (1974); Borell (1975); Ledoux (1996); Talagrand (1996) and references therein. A general review of a certain number of classical results is in Adler (1990, 2000).

2. Describing the behaviour of $F(T, u)$ under various asymptotics : Qualls and Watanabe (1973); Piterbarg (1981, 1996); Leadbetter, Lingren and Rootzén (1983); Berman (1985a, b, 1992); Talagrand (1988); Berman \& Kôno (1992) ; Sun (1993); Wschebor (2000); Azaïs, Bardet and Wschebor (2000). 
3. Studying the regularity of the distribution of $M_{T}$ : Ylvisaker (1968); Tsirelson (1975); Weber (1985); Lifshits (1995); Diebolt and Posse (1996); Azaïs and Wschebor (2000) and references therein.

Generally speaking, even though important results are associated with problems 1) 2) and 3) they only give limited answers to the computation of $F(T, u)$ for fixed $T$ and $u$. As a consequence, Monte-Carlo methods based on the simulation of the paths of the continuous parameter process $X$ are widely used, even though they have well-known difficulties : a) they are poor for theoretical purposes ; b) they are expensive from the point of view of the number of elementary computations needed to assure that the error is below a given bound and c) they always depend on the quality of the random number generator being employed.

The approach in this paper is based upon expressing $F(T, u)$ by means of a series (The "Rice series") whose terms contain the factorial moments of the number of upcrossings. The underlying ideas have been known since a long time (Rice (1944-1945), Slepian (1962), Miroshin (1974)).

The main new result in this paper is that we have been able to prove the convergence of that series in a general framework instead of considering only some particular processes. This provides a method that can be widely applied.

A typical application is Theorem2.2 that states that if a stationary Gaussian process has a covariance which has a Taylor expansion at zero that is absolutely convergent at $t=2 T$, then $F(T, u)$ can be computed by means of the Rice series. On the other hand, even though Theorems 2.1 and 2.3 below do not refer specifically to Gaussian processes, in practice, at present we are only able to apply them to the numerical computation of $F(T, u)$ only in Gaussian cases.

In the section "Numerical examples" we include a comparison between the complexities of the computations of $F(T, u)$ using the Rice series versus Monte-Carlo method, in the relevant case of a general class of stationary Gaussian processes. It shows that the use of Rice series is a priori better. More important is the fact that the Rice series is self-controlling for the numerical errors. This implies that the a posteriori number of computations may be much smaller than the one required by simulation. In fact, in relevant cases for standard bounds for the error, the actual computation is performed with a few terms in the Rice series.

As examples we give tables for $F(T, u)$ for a number of Gaussian processes. When the length of the interval $T$ increases, one needs an increasing mumber of terms in the Rice's series not to surpass a given bound for the approximation error. For small values of $T$ an large values of the level $u$ one can use the so- 
called "Davies bound"(1977), or more accurately, the first term in the Rice series to obtain approximations for $F(T, u)$. But as $T$ increases, for moderate values of $u$ the "Davies bound" is far from the true value and one requires the computation of the succesive terms. The numerical results are shown in the case of four Gaussian stationary processes for which no closed formula is known.

An asymptotic approximation of $F(T, u)$ as $u \rightarrow+\infty$ recently obtained by Azaïs, Bardet and Wschebor (2000). It extends to any $T$ a previous result by Piterbarg (1981) for sufficiently small $T$.

One of the key points in the computation is the numerical approximation of the factorial moments of upcrossings by means of Rice' integral formulae. For that purpose, the main difficulty is the precise description of the behaviour of the integrands appearing in these formulae near the diagonal, which is again an old subject that is interesting on its own - see Belayeiv (1966), Cuzick (1975) - and remains widely open. We have included in the Section "Computation of Moments" some new results, that give partial answers and are helpful to improve the numerical methods.

The extension to processes with non-smooth trajectories can be done by smoothing the paths by means of a deterministic device, applying the previous methods to the regularized process and estimating the error as a function of the smoothing width. We have not included these type of results here since for the time being they do not appear to be of practical use.

The Note (Azaïs \& Wschebor 1997) contains a part of the results in the present paper, without proofs.

\section{Notations}

Let $f: I \longrightarrow \mathbb{R}$ be a function defined on the interval $I$ of the real numbers,

$$
\begin{gathered}
C_{u}(f ; I):=\{t \in I: f(t)=u\} \\
N_{u}(f ; I):=\sharp\left(C_{u}(f ; I)\right)
\end{gathered}
$$

denote respectively the set of roots of the equation $f(t)=u$ on the interval $I$ and the number of these roots, with the convention $N_{u}(f ; I)=+\infty$ if the set $C_{u}$ is infinite. $N_{u}(f ; I)$ is called the number of "crossings" of $f$ with the "level" $u$ on the interval $I$. In what follows, $I$ will be the interval $[0, T]$ if it is not stated otherwise.

In the same way, if $f$ is a differentiable function the number of "upcrossings" of $f$ is defined by means of

$$
U_{u}(f ; I):=\sharp\left(\left\{t \in I: f(t)=u, f^{\prime}(t)>0\right\}\right) .
$$


$\|f\|_{p}$ denotes the norm of $f$ in $L^{p}(I, \lambda), 1 \leq p \leq+\infty, \lambda$ the Lebesgue measure. The joint density of the finite set of random variables $X_{1}, \ldots X_{n}$ at the point $\left(x_{1}, \ldots x_{n}\right)$ will be denoted $p_{X_{1}, \ldots, X_{n}}\left(x_{1}, \ldots x_{n}\right)$ whenever it exists. $\phi(t):=(2 \pi)^{-1 / 2} \exp \left(-t^{2} / 2\right)$ is the density of the standard normal distribution, $\Phi(t):=\int_{\infty}^{t} \phi(u) d u$ its distribution function. $|I|$ is the length of $I . x^{+}=\sup \{x, 0\}$.

If $A$ is a matrix, $A^{T}$ denotes its transposed, and if $A$ is a square matrix, $\operatorname{det}(A)$ its determinant. $\operatorname{Var}(\xi)$ is the variance matrix of the (finite dimensional) random vector $\xi$ and $\operatorname{Cov}(\xi, \eta)$ the covariance of $\xi$ and $\eta$.

For $m$ and $k$, positive integers, $k \leq m$, define the factorial $k t h$ power of $m$ by

$$
m^{[k]}:=m(m-1) \ldots(m-k+1)
$$

For other real values of $m$ and $k$ we put $m^{[k]}:=0$.

If $k$ is an integer $k \geq 1$, the "diagonal of $I^{k}$ " is the set:

$$
\mathcal{D}_{k}(I):=\left\{\left(t_{1}, \ldots, t_{k}\right) \in I^{k}, t_{j}=t_{h} \text { for some pair }(j, h), j \neq h\right\} .
$$

$f^{(m)}$ is the $m$-th derivative of the function $f . \delta_{j h}=0$ or 1 according as $j \neq h$ or $j=h$.

\section{The distribution of the maximum and the Rice series}

We introduce the notations

$$
\nu_{m}:=E\left(\left(U_{u}\right)^{[m]} \mathbb{I}_{\left\{X_{0} \leq u\right\}}\right) ; \quad \widetilde{\nu}_{m}:=E\left(\left(U_{u}\right)^{[m]}\right)(m=1,2, \ldots)
$$

where $U_{u}=U_{u}(X,[0, T]) . \nu_{m}$ is the factorial moment of the number of upcrossings of the process $X$ with the level $u$ on the interval $[0, T]$, starting below $u$ at $t=0$.

The Rice formula to compute $\nu_{m}$, whenever it holds is:

$$
\begin{aligned}
\nu_{m}= & \int_{[0, T]^{m}} d t_{1} \ldots d t_{m} \int_{-\infty}^{u} d x E\left(\left(X_{t_{1}}^{\prime}\right)^{+} \ldots\left(X_{t_{m}}^{\prime}\right)^{+} / X_{0}=x, X_{t_{1}}=\ldots=X_{t_{m}}=u\right) \\
& p_{X_{0}, X_{t_{1}}, \ldots, X_{t_{m}}}(x, u, \ldots, u)= \\
= & \int_{[0, T]^{m}} d t_{1} \ldots d t_{m} \int_{-\infty}^{u} d x \int_{[0,+\infty)^{m}} x_{1}^{\prime} \ldots x_{m}^{\prime} \\
& p_{X_{0}, X_{t_{1}}, \ldots, X_{t_{m}}, X_{t_{1}}^{\prime}, \ldots, X_{t_{m}}^{\prime}}\left(x, u, \ldots, u, x_{1}^{\prime}, \ldots x_{m}^{\prime}\right) d x_{1}^{\prime} \ldots d x_{m}^{\prime} .
\end{aligned}
$$


(References for conditions for this formula to hold true that suffice for our presente purposes and also for proofs can be found, for example, in Marcus (1977) and in Wschebor (1985).

This section contains two main results. The first is Theorem 2.1 that requires the process to have $\mathcal{C}^{\infty}$ paths and contains a general condition enabling to compute $F(T, u)$ as the sum of a series. The second is Theorem 2.2 that illustrates the same situation for Gaussian stationary processes from conditions on the the covariance. As for Theorem 2.3, it contains upper and lower bounds on $F(T, u)$ for processes with $C^{k}$-paths verifying some additional conditions.

Theorem 2.1 Assume that a.s. the paths of the stochastic process $X$ are of class $\mathcal{C}^{\infty}$ and that the density $p_{X_{T / 2}}$ is bounded by some constant $D$.

(i) If there exists a sequence of positive numbers $\left\{c_{k}\right\}_{k=1,2, \ldots}$ such that:

$$
\gamma_{k}:=P\left(\left\|X^{(2 k-1)}\right\|_{\infty} \geq c_{k} \cdot T^{2 k-1}\right)+\frac{D c_{k}}{2^{2 k-1}(2 k-1) !}=o\left(2^{-k}\right)(k \rightarrow \infty)
$$

then:

$$
1-F(T, u)=P\left(X_{0}>u\right)+\sum_{m=1}^{\infty}(-1)^{m+1} \frac{\nu_{m}}{m !}
$$

(ii) In formula (4) the error when one replaces the infinite sum by its $m_{0}$-th partial sum is bounded by $\gamma_{m_{0}+1}^{*}$ where:

$$
\gamma_{m}^{*}:=\sup _{k \geq m}\left(2^{k+1} \gamma_{k}\right)
$$

We will call the series in the right-hand term of (4) the "Rice Series".

For the proof we will assume, with no loss of generality that $T=1$.

We start with the following lemma on the Cauchy remainder for polynomial interpolation (Davis 1975, Th. 3.1.1 ).

Lemma 2.1 a) Let $I$ be an interval in the real line, $f: I \longrightarrow \mathbb{R}$ a function of class $\mathcal{C}^{k}, k$ a positive integer, $t_{1}, \ldots, t_{k}, k$ points in $I$ and let $P(t)$ be the - unique - interpolation polynomial of degree $k-1$ such that for $i=1, \ldots, k: f\left(t_{i}\right)=P\left(t_{i}\right)$, taking into account possible multiplicities. 
Then, for $t \in I$ :

$$
f(t)-P(t)=\frac{1}{k !}\left(t-t_{1}\right) \ldots\left(t-t_{k}\right) f^{(k)}(\xi)
$$

where

$$
\min \left(t_{1}, \ldots, t_{k}, t\right) \leq \xi \leq \max \left(t_{1}, \ldots, t_{k}, t\right) .
$$

b) If $f$ is of class $\mathcal{C}^{k}$ and has $k$ zeros in I (taking into account possible multiplicities), then:

$$
|f(1 / 2)| \leq \frac{1}{k ! 2^{k}}\left\|f^{(k)}\right\|_{\infty}
$$

The next combinatorial lemma plays the central role in what follows. A proof is given in Lindgren (1972).

Lemma 2.2 Let $\xi$ be a non-negative integer-valued random variable having finite moments of all orders. Let $k, m, M(k \geq 0, m \geq 1, M \geq 1)$ be integers and denote

$$
p_{k}:=P(\xi=k) \quad ; \quad \mu_{m}:=E\left(\xi^{[m]}\right) \quad ; \quad S_{M}:=\sum_{m=1}^{M}(-1)^{m+1} \frac{\mu_{m}}{m !}
$$

Then

(i) For each $M$ :

$$
S_{2 M} \leq \sum_{k=1}^{2 M} p_{k} \leq \sum_{k=1}^{\infty} p_{k} \leq S_{2 M+1}
$$

(ii) The sequence $\left\{S_{M} ; M=1,2, \ldots\right\}$ has a finite limit if and only if $\mu_{m} / m$ ! $\rightarrow 0$ as $m \rightarrow \infty$, and in that case:

$$
P(\xi \geq 1)=\sum_{k=1}^{\infty} p_{k}=\sum_{m=1}^{\infty}(-1)^{m+1} \frac{\mu_{m}}{m !} .
$$

Remark. A by-product of Lemma 2.2 that will be used in the sequel is the following: if in (6) one substitutes the infinite sum by the $M$-partial sum, the absolute value $\mu_{M+1} /((M+1) !)$ of the first neglected term is an upper-bound for the error in the computation of $P(\xi \geq 1)$. 
Lemma 2.3 With the same notations as in Lemma 2.2 we have the equality:

$$
E\left(\xi^{[m]}\right)=m \sum_{k=m}^{\infty}(k-1)^{[m-1]} P(\xi \geq k) \quad(m=1,2, \ldots) .
$$

Proof: Check the identity

$$
j^{[m]}=m \sum_{k=m-1}^{j-1}(k)^{[m-1]}
$$

for each pair of integers $j, m$. So,

$$
\begin{gathered}
E\left(\xi^{[m]}\right)=\sum_{j=m}^{\infty} j^{[m]} P(\xi=j)=\sum_{j=m}^{\infty} P(\xi=j) m \sum_{k=m}^{j}(k-1)^{[m-1]}= \\
=m \sum_{k=m}^{\infty}(k-1)^{[m-1]} P(\xi \geq k) .
\end{gathered}
$$

Lemma 2.4 Suppose that a.s. the paths of the process $X$ belong to $\mathcal{C}^{\infty}$ and that $p_{X_{1 / 2}}$ is bounded by the constant $D$. Then for any sequence $\left\{c_{k}, k=1,2, \ldots\right\}$ of positive numbers, one has

$$
E\left(\left(U_{u}\right)^{[m]}\right) \leq m \sum_{k=m}^{\infty}(k-1)^{[m-1]}\left[P\left(\left\|X^{(2 k-1)}\right\|_{\infty} \geq c_{k}\right)+\frac{D c_{k}}{2^{2 k-1}(2 k-1) !}\right]
$$

Proof: Because of Lemma 2.3 it is enough to prove that $P\left(U_{u} \geq k\right)$ is bounded by the expression in brackets in the right-hand member of (7). We have

$$
P\left(U_{u} \geq k\right) \leq P\left(\left\|X^{(2 k-1)}\right\|_{\infty} \geq c_{k}\right)+P\left(U_{u} \geq k,\left\|X^{(2 k-1)}\right\|_{\infty}<c_{k}\right) .
$$

Because of Rolle's theorem:

$$
\left\{U_{u} \geq k\right\} \subset\left\{N_{u}(X ; I) \geq 2 k-1\right\},
$$

Applying Lemma 2.1 to the function $X_{(.)}-u$ and replacing in its statement $k$ by $2 k-1$, we obtain:

$$
\left\{U_{u} \geq k,\left\|X^{(2 k-1)}\right\|_{\infty}<c_{k}\right\} \subset\left\{\left|X_{1 / 2}-u\right| \leq \frac{c_{k}}{2^{2 k-1}(2 k-1) !}\right\} .
$$


The remaining is plain.

Proof of Theorem 2.1:

We use the notation $\tilde{\nu}_{m}:=E\left(U_{u}^{[m]}\right) \quad(m=1,2, \ldots)$.

Using Lemma 2.4 and the hypothesis we obtain:

$$
\frac{\tilde{\nu}_{m}}{m !} \leq \frac{1}{m !} \sum_{k=m}^{\infty} k^{[m]} \gamma_{m}^{*} 2^{-(k+1)}=\frac{\gamma_{m}^{*}}{m !} 2^{-(m+1)}\left[\left.\left(\frac{1}{1-x}\right)^{(m)}\right|_{x=1 / 2}\right]=\gamma_{m}^{*}
$$

Since $\nu_{m} \leq \tilde{\nu}_{m}$ we can apply Lemma 2.2 to the random variable $\xi=U_{u} \mathbb{I}_{\left\{X_{0} \leq u\right\}}$ and the result follows from $\gamma_{m}^{*} \rightarrow 0$.

\section{Remarks}

One can replace condition $p_{X_{T / 2}}(x) \leq D$ for all $x$ by $p_{X_{T / 2}}(x) \leq D$ for $x$ in some neighbourhood of $u$. In this case, the statement of Theorem 2.1 holds if one adds in (ii) that the error is bounded by $\gamma_{m_{0}+1}^{*}$ for $m_{0}$ large enough. The proof is similar. Also, one may substitute the one-dimensional density $p_{X_{T / 2}}$ by $p_{X_{t}}$ for some other $t \in(0, T)$, introducing into the bounds the corresponding modifications.

The application of Theorem 2.1 requires an adequate choice of the sequence $\left\{c_{k}, k=1,2, \ldots\right\}$ that depends on the available description of the process $X$. The whole procedure will have some practical interest for the computation of $P(M>u)$ only if we get appropriate bounds for the quantities $\gamma_{m}^{*}$ and the factorial moments $\nu_{m}$ can be actually computed by means of Rice formulae (or by some other procedure). The next Theorem shows how this can be done in the case of a general class of Gaussian stationary processes.

Theorem 2.2 Let $X$ be Gaussian, centered and stationary, with covariance $\Gamma$.

Assume that $\Gamma$ has a Taylor expansion at the origin that is absolutely convergent at $t=2 T$. Then the conclusion of Theorem 2.1 holds true so that the Rice series converges and $F(T, u)$ can be computed by means of (4)

Proof. Again we assume with no loss of generality that $T=1$ and that $\Gamma(0)=1$.

Note that the hypothesis implies that the spectral moments $\lambda_{k}$ exist and are finite for every $k=0,1,2, \ldots$

We will prove a stronger result, assuming the hypothesis:

$$
H_{1}: \lambda_{2 k} \leq C_{1}(k !)^{2}
$$


It is easy to verify that if $\Gamma$ has a Taylor expansion at zero that is absolutely convergent at $t=2$, then $H_{1}$ holds true. (In fact, both conditions are only slightly different, since $H_{1}$ implies that the Taylor expansion of $\Gamma$ at zero is absolutely convergent in $\{|t|<2\}$ ).

Let us check that the hypotheses of Theorem 2.1 hold true.

First, $p_{X_{1 / 2}}(x) \leq D=(2 \pi)^{-1 / 2}$.

Second, let us show a sequence $\left\{c_{k}\right\}$ that satisfies (3). We have

$$
\begin{aligned}
P\left(\left\|X^{(2 k-1)}\right\|_{\infty} \geq c_{k}\right) \leq P\left(\left|X_{0}^{(2 k-1)}\right|\right. & \left.\geq c_{k}\right)+2 P\left(U_{c_{k}}\left(X^{(2 k-1)}, I\right) \geq 1\right) \leq \\
& \leq P\left(|Z| \geq c_{k} \lambda_{4 k-2}^{-1 / 2}\right)+2 E\left(U_{c_{k}}\left(X^{(2 k-1)}, I\right)\right),
\end{aligned}
$$

where $Z$ is standard normal.

Note that $\left\{X_{t}^{(2 k-1)} ; t \in \mathbb{R}\right\}$ is a Gaussian stationary centered process with covariance function $-\Gamma^{(4 k-2)}($.$) . So we can use Rice formula for the expectation of the$ number of upcrossings of a stationary centered Gaussian process (see for example Cramér \& Leadbetter, 1967) to compute the second term in the right-hand member of (8). Using the inequality $1-\Phi(x) \leq(1 / x) \phi(x)$ valid for $x>0$, one gets:

$$
P\left(\left\|X^{(2 k-1)}\right\|_{\infty} \geq c_{k}\right) \leq\left[\sqrt{\frac{2}{\pi}} \frac{\lambda_{4 k-2}^{1 / 2}}{c_{k}}+(1 / \pi)\left(\frac{\lambda_{4 k}}{\lambda_{4 k-2}}\right)^{1 / 2}\right] \exp \left(-\frac{c_{k}^{2}}{2 \lambda_{4 k-2}}\right)
$$

Choose

$$
\begin{gathered}
c_{k}:=\left(B_{1} k \lambda_{4 k-2}\right)^{1 / 2} \text { if } \frac{\lambda_{4 k}}{\lambda_{4 k-2}} \leq B_{1} k \\
c_{k}:=\left(\lambda_{4 k}\right)^{1 / 2} \text { if } \frac{\lambda_{4 k}}{\lambda_{4 k-2}}>B_{1} k .
\end{gathered}
$$

Using hypothesis $H_{1}$ ), if $B_{1}>1$ :

$$
P\left(\left\|X^{(2 k-1)}\right\|_{\infty} \geq c_{k}\right) \leq\left[\sqrt{\frac{2}{\pi}}+\frac{1}{\pi}\left(B_{1} k\right)^{1 / 2}\right] e^{-\frac{B_{1} k}{2}} .
$$

Finally, choosing $B_{1}:=4 \log (2)$ :

$$
\gamma_{k} \leq \sqrt{\frac{2}{\pi}}\left(1+2\left(C_{1}^{1 / 2}+1\right) k\right) 2^{-2 k} \quad(k=1,2, \ldots)
$$


so that (3) is satisfied. As a by product, note that

$$
\gamma_{m}^{*} \leq \sqrt{\frac{8}{\pi}}\left(1+2\left(C_{1}^{1 / 2}+1\right) m\right) 2^{-m} \quad(m=1,2, \ldots) .
$$

\section{Remarks}

a) If one is willing to use Rice formulae to compute the factorial moments $\nu_{m}$, it is enough to verify that the distribution of

$$
X_{t_{1}}, \ldots, X_{t_{k}}, X_{t_{1}}^{\prime}, \ldots, X_{t_{k}}^{\prime}
$$

is non-degenerate for any choice of $k=1,2, \ldots\left(t_{1}, \ldots, t_{k}\right) \in I^{k} \backslash \mathcal{D}_{k}(I)$. For Gaussian stationary processes a sufficient condition for non-degeneracy is the spectral measure not to be purely atomic (see Cramér and Leadbetter (1967) for a proof). The same kind of argument permits to show that the conclusion remains if the spectral measure is purely atomic and the set of its atoms has an acumulation point in $\mathbb{R}$. Sufficient conditions for the finiteness of $\nu_{m}$ are given also in Nualart \& Wschebor (Lemma $1.2,1991)$.

b) If instead of requiring the paths of the process $X$ to be of class $\mathcal{C}^{\infty}$, one relaxes this condition up to a certain order of differentiability, one can still get upper and lower bounds for $P(M>u)$.

Theorem 2.3 Let $X=\left\{X_{t}: t \in I\right\}$ be a real-valued stochastic process. Suppose that $p_{X_{t}}(x)$ is bounded for $t \in I, x \in \mathbb{R}$ and that the paths of $X$ are of class $\mathcal{C}^{p+1}$. Then

$$
\text { if } 2 K+1<p / 2: P(M>u) \leq P\left(X_{0}>u\right)+\sum_{m=1}^{2 K+1}(-1)^{m+1} \frac{\nu_{m}}{m !}
$$

and

$$
\text { if } \quad 2 K<p / 2: P(M>u) \leq P\left(X_{0}>u\right)+\sum_{m=1}^{2 K}(-1)^{m+1} \frac{\nu_{m}}{m !} .
$$

Note that all the moments in the above formulae are finite.

The proof is a straightforward application of Lemma 2.2 and Lemma 1.2 in Nualart \& Wschebor (1991).

When the level $u$ is high, the results by Piterbag $(1981,1996)$, which were until recently the sharpest known asymptotic bounds for the tail of the distribution of the 
maximum on a fixed interval of general Gaussian stationary processes with regular paths (for a refinement, see Azaïs, Bardet and Wschebor, 2000) can be deduced from the foregoing arguments. Here, only the first term in the Rice series takes part in the equivalent of $P(M>u)$ as $u \rightarrow+\infty$. More precisely, if $\lambda_{4}<\infty$, it is not hard to prove that

$$
\begin{gathered}
0 \leq \sqrt{\frac{\lambda_{2}}{2 \pi}} \phi(u)-\nu_{1} \leq(\text { const }) e^{\frac{-u^{2}(1+\eta)}{2}}, \\
\nu_{2} \leq(\text { const }) e^{-\frac{u^{2}(1+\eta)}{2}},
\end{gathered}
$$

for a certain $\eta>0$. Lemma 3.2 implies that

$$
0 \leq 1-\Phi(u)+\sqrt{\frac{\lambda_{2}}{2 \pi}} \phi(u)-P(M>u) \leq(\text { const }) e^{-\frac{u^{2}(1+\eta)}{2}},
$$

which is Piterbarg's result.

\section{Computation of Moments}

An efficient numerical computation of the factorial moments of crossings is associated to a fine description of the behaviour as the $k$-tuple $\left(t_{1}, \ldots, t_{k}\right)$ approaches the diagonal $\mathcal{D}_{k}(I)$, of the integrands

$$
\begin{gathered}
\widetilde{A}_{t_{1}, \ldots, t_{k}}^{+}(u, \ldots, u)=\int_{[0,+\infty)^{m}} x_{1}^{\prime} \ldots x_{m}^{\prime} p_{X_{t_{1}}, \ldots, X_{t_{m}}, X_{t_{1}}^{\prime}, \ldots, X_{t_{m}}^{\prime}}\left(u, \ldots, u, x_{1}^{\prime}, \ldots x_{m}^{\prime}\right) d x_{1}^{\prime} \ldots d x_{m}^{\prime} . \\
A_{t_{1}, \ldots, t_{k}}(u)=\int_{-\infty}^{u} d x \int_{[0,+\infty)^{m}} x_{1}^{\prime} \ldots x_{m}^{\prime} p_{X_{0}, X_{t_{1}}, \ldots, X_{t_{m}}, X_{t_{1}}^{\prime}, \ldots, X_{t_{m}}^{\prime}}\left(x, u, \ldots, u, x_{1}^{\prime}, \ldots x_{m}^{\prime}\right) d x_{1}^{\prime} \ldots d x_{m}^{\prime} .
\end{gathered}
$$

that appear respectively in Rice formulae for the $k^{\text {th }}$-factorial moment of upcrossings or the $k^{\text {th }}$-factorial moment of upcrossings with the additional condition that $X_{0} \leq u$ (see formula $(2)$.

For example in Azaïs, Cierco and Croquette (1999) it is proved that if $X$ is Gaussian, stationary, centered and $\lambda_{8}<\infty$, then the integrand $\widetilde{A}_{s, t}^{+}(u, u)$ in the 
computation of $\tilde{\nu}_{2}$ - the second factorial moment of the number of upcrossings satisfies:

$$
\widetilde{A}_{s, t}^{+}(u, u) \simeq \frac{1}{1296} \frac{\left(\lambda_{2} \lambda_{6}-\lambda_{4}^{2}\right)^{3 / 2}}{\left(\lambda_{4}-\lambda_{2}^{2}\right)^{1 / 2} \pi^{2} \lambda_{2}^{2}} \exp \left(-\frac{1}{2} \frac{\lambda_{4}}{\lambda_{4}-\lambda_{2}^{2}} u^{2}\right)(t-s)^{4},
$$

as $t-s \rightarrow 0$.

(12) can be extended to non-stationary Gaussian processes obtaining an equivalence of the form:

$$
\widetilde{A}_{s, t}^{+}(u, u) \simeq J(\widetilde{t})(t-s)^{4} \quad \text { as } \quad s, t \rightarrow \widetilde{t}
$$

where $J(\widetilde{t})$ is a continuous non-zero function of $\widetilde{t}$ depending on $u$, that can be expressed in terms of the mean and covariance functions of the process and its derivatives. We give a proof of an equivalence of the form (13) in the next proposition.

One can profit of this equivalence to improve the numerical methods to compute $\nu_{2}$ (the second factorial moment of the number of upcrossings restricted to $X_{0} \leq$ $u$ ). Equivalence formulae such as (12) or (13) can be used to avoid numerical degeneracies near the diagonal $D_{2}(I)$. Note that even in case $X$ is stationary at the departure, under conditioning on $X_{0}$, the process that must be taken into account in the actual computation of the factorial moments of upcrossings that appear in the Rice series(4) will be non-stationary, so that equivalence (13) is the appropriate tool.

Proposition 3.1 Suppose that $X$ is a Gaussian process with $C^{5}$ paths and that for each $t \in I$ the joint distribution of $X_{t}, X_{t}^{\prime}, X_{t}^{(2)}, X_{t}^{(3)}$ does not degenerate.Then (13) holds true.

Proof. Denote by $\xi=\left(\begin{array}{l}\xi_{1} \\ \xi_{2}\end{array}\right)$ a two-dimensional random vector having as probability distribution the conditional distribution of $\left(\begin{array}{c}X_{s}^{\prime} \\ X_{t}^{\prime}\end{array}\right)$ given $X_{s}=X_{t}=u$.

One has:

$$
\widetilde{A}_{s, t}^{+}(u, u)=E\left(\xi_{1}^{+} \xi_{2}^{+}\right) p_{X_{s}, X_{t}}(u, u)
$$

Put $\tau=t-s$ and check the following Taylor expansions around the point $s$ :

$$
\begin{gathered}
E\left(\xi_{1}\right)=m_{1} \tau+m_{2} \tau^{2}+L_{1} \tau^{3} \\
E\left(\xi_{2}\right)=-m_{1} \tau+m_{2}^{\prime} \tau^{2}+L_{2} \tau^{3}
\end{gathered}
$$




$$
\operatorname{Var}(\xi)=\left(\begin{array}{lr}
a \tau^{2}+b \tau^{3}+c \tau^{4}+\rho_{11} \tau^{5} & -a \tau^{2}-\frac{b+b^{\prime}}{2} \tau^{3}+d \tau^{4}+\rho_{12} \tau^{5} \\
-a \tau^{2}-\frac{b+b^{\prime}}{2} \tau^{3}+d \tau^{4}+\rho_{12} \tau^{5} & a \tau^{2}+b^{\prime} \tau^{3}+c^{\prime} \tau^{4}+\rho_{22} \tau^{5}
\end{array}\right)
$$

where $m_{1}, m_{2}, m_{2}^{\prime}, a, b, c, d, a^{\prime}, b^{\prime}, c^{\prime}$ are continuous functions of $s$ and $L_{1}, L_{2}, \rho_{11}$, $\rho_{12}, \rho_{22}$ are bounded functions of $s$ and $t$. (15),(16) and (17) follow directly from the regression formulae of the pair $\left(\begin{array}{l}X_{s}^{\prime} \\ X_{t}^{\prime}\end{array}\right)$ on the condition $X_{s}=X_{t}=u$.

Note that (as in Belyaiev, 1966 or Azaïs and Wschebor, 2000)

$$
\operatorname{Var}\left(\xi_{1}\right)=\frac{\operatorname{det} \operatorname{Var}\left(X_{s}, X_{t}, X_{s}^{\prime}\right)^{T}}{\operatorname{det} \operatorname{Var}\left(X_{s}, X_{t}\right)^{T}}=\frac{\operatorname{det} \operatorname{Var}\left(X_{s}, X_{s}^{\prime}, X_{t}-X_{s}-(t-s) X_{s}^{\prime}\right)^{T}}{\operatorname{det} \operatorname{Var}\left(X_{s}, X_{t}-X_{s}\right)^{T}}
$$

A direct computation gives:

$$
\begin{gathered}
\operatorname{det} \operatorname{Var}\left(X_{s}, X_{t}\right)^{T} \approx \tau^{2} \operatorname{det} \operatorname{Var}\left(X_{s}, X_{s}^{\prime}\right)^{T} \\
\operatorname{Var}\left(\xi_{1}\right) \approx \frac{1}{4} \frac{\operatorname{det} \operatorname{Var}\left(X_{s}, X_{s}^{\prime}, X_{s}^{(2)}\right)^{T}}{\operatorname{det} \operatorname{Var}\left(X_{s}, X_{s}^{\prime}\right)^{T}} \tau^{2}
\end{gathered}
$$

where $\approx$ denotes equivalence as $\tau \rightarrow 0$. So,

$$
a=\frac{1}{4} \frac{\operatorname{det} \operatorname{Var}\left(X_{s}, X_{s}^{\prime}, X_{s}^{(2)}\right)^{T}}{\operatorname{det} \operatorname{Var}\left(X_{s}, X_{s}^{\prime}\right)^{T}}
$$

which is a continuous non-vanishing function for $s \in I$. Note that the coefficient of $\tau^{3}$ in the Taylor expansion of $\operatorname{Cov}\left(\xi_{1}, \xi_{2}\right)$ is equal to $-\frac{b+b^{\prime}}{2}$. This follows either by direct computation or noting that det $\operatorname{Var}(\xi)$ is a symmetric function of the pair $s, t$.

Put

$$
\Delta(s, t)=\operatorname{det} \operatorname{Var}(\xi)
$$

The behaviour of $\Delta(s, t)$ as $s, t \rightarrow \widetilde{t}$ can be obtained by noting that

$$
\Delta(s, t)=\frac{\operatorname{det} \operatorname{Var}\left(X_{s}, X_{t}, X_{s}^{\prime}, X_{t}^{\prime}\right)^{T}}{\operatorname{det} \operatorname{Var}\left(X_{s}, X_{t}\right)^{T}}
$$

and applying Lemma 3.2 in Azaïs and Wschebor (2000) or Lemma 4.3, p.76 in Piterbarg (1996) which provide an equivalent for the numerator, so that:

$$
\Delta(s, t) \approx \bar{\Delta}(\widetilde{t}) \tau^{6}
$$


with

$$
\bar{\Delta}(\widetilde{t})=\frac{1}{144} \frac{\operatorname{det} \operatorname{Var}\left(X_{\widetilde{t}}, X_{\widetilde{t}}^{\prime}, X_{\widetilde{t}}^{(2)}, X_{\widetilde{t}}^{(3)}\right)^{T}}{\operatorname{det} \operatorname{Var}\left(X_{\widetilde{t}}, X_{\widetilde{t}}^{\prime}\right)^{T}}
$$

The non degeneracy hypothesis implies that $\bar{\Delta}(\widetilde{t})$ is continuous and non zero.

Then:

$$
E\left(\xi_{1}^{+} \xi_{2}^{+}\right)=\frac{1}{2 \pi[\Delta(s, t)]^{1 / 2}} \int_{0}^{+\infty} \int_{0}^{+\infty} x y \exp \left[-\frac{1}{2 \Delta(s, t)} F(x, y)\right] d x d y
$$

where

$F(x, y)=\operatorname{Var}\left(\xi_{2}\right)\left(x-E\left(\xi_{1}\right)\right)^{2}+\operatorname{Var}\left(\xi_{1}\right)\left(y-E\left(\xi_{2}\right)\right)^{2}-2 \operatorname{Cov}\left(\xi_{1}, \xi_{2}\right)\left(x-E\left(\xi_{1}\right)\right)\left(y-E\left(\xi_{2}\right)\right)$

Substituting the expansions (15), (16), (17) in the integrand of (20) and making the change of variables $x=\tau^{2} v, y=\tau^{2} w$ we get, as $s, t \rightarrow \widetilde{t}$ :

$$
E\left(\xi_{1}^{+} \xi_{2}^{+}\right) \approx \frac{\tau^{5}}{2 \pi[\bar{\Delta}(\widetilde{t})]^{1 / 2}} \int_{0}^{+\infty} \int_{0}^{+\infty} v w \exp \left[-\frac{1}{2 \bar{\Delta}(\widetilde{t})} \bar{F}(v, w)\right] d v d w
$$

$\bar{\Delta}(\widetilde{t})$ can also be expressed in terms of the functions $a, b, c, d, a^{\prime}, b^{\prime}, c^{\prime}$ :

$$
\bar{\Delta}(\widetilde{t})=a c^{\prime}+c a^{\prime}+2 a d-\left(\frac{b-b^{\prime}}{2}\right)^{2}
$$

and

$$
\bar{F}(v, w)=a\left(v-m_{2}+w-m_{2}^{\prime}\right)^{2}+m_{1}^{2}\left(c+c^{\prime}+2 d\right)-m_{1}\left(b-b^{\prime}\right)\left(v+w-m_{2}-m_{2}^{\prime}\right)
$$

The functions $a, b, c, d, b^{\prime}, c^{\prime}, m_{1}, m_{2}$ that appear in these formulae are all evaluated at the point $\widetilde{t}$.

Replacing (21) and (18) into (14) one gets (13).

For $k \geq 3$, the general behaviour of the functions $A_{t_{1}, \ldots, t_{k}}(u)$ and $\widetilde{A}_{t_{1}, \ldots, t_{k}}^{+}(u, \ldots, u)$ when $\left(t_{1}, \ldots, t_{k}\right)$ approaches the diagonal is not known. Proposition 3.3 , even though it contains restrictive conditions (it requires $E\left\{X_{t}\right\}=0$ and $u=0$ ) can be applied to improve the efficiency in the computation of the $k^{\text {th }}$-factorial moments by means of a Monte-Carlo method, via the use of important sampling. More precisely, when 
computing the integral of $\widetilde{A}_{t_{1}, \ldots, t_{k}}^{+}(u)$ over $I^{k}$, instead of choosing at random the point $\left(t_{1}, t_{2}, \ldots, t_{k}\right)$ in the cube $I^{k}$ with a uniform distribution, we do it with a probability law that has a density proportional to the function $\prod_{1 \leq i<j \leq k}\left(t_{j}-t_{i}\right)^{4}$. For its proof we will use the following auxiliary proposition, that has its own interest and extends (19) to any k.

Proposition 3.2 Suppose that $X=\left\{X_{t}: t \in I\right\}$ is a Gaussian process defined on the interval $I$ of the real line with $C^{2 k-1}$ paths, $k$ an integer, $k \geq 2$, and that the joint distribution of $\left(X_{t}, X_{t}^{\prime}, \ldots, X_{t}^{(2 k-1)}\right)$ is non-degenerate for each $t \in I$. Then, if $t_{1}, t_{2}, \ldots, t_{k} \rightarrow t^{*}:$

$\Delta=\operatorname{det} \operatorname{Var}\left(X_{t_{1}}, X_{t_{1}}^{\prime}, \ldots, X_{t_{k}}, X_{t_{k}}^{\prime}\right)^{T} \approx \frac{\operatorname{det} \operatorname{Var}\left(X_{t^{*}}, X_{t^{*}}^{\prime}, \ldots, X_{t^{*}}^{(2 k-1)}\right)^{T}}{[2 ! .3 ! \ldots . .(2 k-1) !]^{2}} \prod_{1 \leq i<j \leq k}\left(t_{j}-t_{i}\right)^{8}$

Proof. With no loss of generality, we consider only $k$-tuples $\left(t_{1}, t_{2}, \ldots, t_{k}\right)$ such that $t_{i} \neq t_{j}$ if $i \neq j$.

Suppose $f: I \longrightarrow \mathbb{R}$ is a function of class $C^{2 m}, m \geq 1$, and $t_{1}, t_{2}, \ldots, t_{m}$ are pairwise different points in $I$. We use the following notations for interpolating polynomials:

$P_{m}(t ; f)$ is the polynomial of degree $2 m-1$ such that

$$
P_{m}\left(t_{j} ; f\right)=f\left(t_{j}\right) \quad \text { and } \quad P_{m}^{\prime}(t ; f)=f^{\prime}\left(t_{j}\right) \quad \text { for } j=1, \ldots, m .
$$

$Q_{m}(t ; f)$ is the polynomial of degree $2 m-2$ such that

$$
Q_{m}\left(t_{j} ; f\right)=f\left(t_{j}\right) \quad \text { for } j=1, \ldots, m ; \quad Q_{m}^{\prime}(t ; f)=f^{\prime}\left(t_{j}\right) \quad \text { for } j=1, \ldots, m-1 .
$$

¿From Lemma 2.1 we know that

$$
\begin{gathered}
f(t)-P_{m}(t ; f)=\frac{1}{(2 m) !}\left(t-t_{1}\right)^{2} \ldots\left(t-t_{m}\right)^{2} f^{(2 m)}(\xi) \\
f(t)-Q_{m}(t ; f)=\frac{1}{(2 m-1) !}\left(t-t_{1}\right)^{2} \ldots .\left(t-t_{m-1}\right)^{2}\left(t-t_{m}\right) f^{(2 m-1)}(\eta)
\end{gathered}
$$

where

$$
\xi=\xi\left(t_{1}, t_{2}, \ldots, t_{m}, t\right), \eta=\eta\left(t_{1}, t_{2}, \ldots, t_{m}, t\right)
$$


and

$$
\min \left(t_{1}, t_{2}, \ldots, t_{m}, t\right) \leq \xi, \eta \leq \max \left(t_{1}, t_{2}, \ldots, t_{m}, t\right) .
$$

Note that the function

$$
g(t)=f^{(2 m-1)}\left(\eta\left(t_{1}, t_{2}, \ldots, t_{m}, t\right)\right)=\frac{(2 m-1) !\left[f(t)-Q_{m}(t ; f)\right]}{\left(t-t_{1}\right)^{2} \ldots .\left(t-t_{m-1}\right)^{2}\left(t-t_{m}\right)}
$$

is differentiable at the point $t=t_{m}$ and differentiating in (24):

$$
f^{\prime}\left(t_{m}\right)-Q_{m}^{\prime}\left(t_{m} ; f\right)=\frac{1}{(2 m-1) !}\left(t_{m}-t_{1}\right)^{2} \ldots\left(t_{m}-t_{m-1}\right)^{2} f^{(2 m-1)}\left(\eta\left(t_{1}, t_{2}, \ldots, t_{m}, t_{m}\right)\right)
$$

Put

$$
\xi_{m}=\xi\left(t_{1}, t_{2}, \ldots, t_{m}, t_{m}\right), \eta_{m}=\eta\left(t_{1}, t_{2}, \ldots, t_{m}, t_{m}\right) .
$$

Since $P_{m}(t ; f)$ is a linear functional of

$$
\left(f\left(t_{1}\right), \ldots, f\left(t_{m}\right), f^{\prime}\left(t_{1}\right), \ldots, f^{\prime}\left(t_{m}\right)\right)
$$

and $Q_{m}(t ; f)$ is a linear functional of

$$
\left(f\left(t_{1}\right), \ldots, f\left(t_{m}\right), f^{\prime}\left(t_{1}\right), \ldots, f^{\prime}\left(t_{m-1}\right)\right)
$$

with coefficients depending (in both cases) only on $t_{1}, t_{2}, \ldots, t_{m}, t$, it follows that:

$$
\begin{gathered}
\Delta=\operatorname{det} \operatorname{Var}\left(X_{t_{1}}, X_{t_{1}}^{\prime}, X_{t_{2}}-P_{1}\left(t_{2} ; X\right), X_{t_{2}}^{\prime}-Q_{2}^{\prime}\left(t_{2}, X\right), \ldots\right. \\
\left.\ldots, X_{t_{k}}-P_{k-1}\left(t_{k} ; X\right), X_{t_{k}}^{\prime}-Q_{k}^{\prime}\left(t_{k} ; X\right)\right)^{T}= \\
=\operatorname{det} \operatorname{Var}\left(X_{t_{1}}, X_{t_{1}}^{\prime}, \frac{1}{2 !}\left(t_{2}-t_{1}\right)^{2} X_{\xi_{1}}^{(2)}, \frac{1}{3 !}\left(t_{2}-t_{1}\right)^{2} X_{\eta_{2}}^{(3)}, \ldots\right. \\
\left.. ., \frac{1}{(2 k-2) !}\left(t_{k}-t_{1}\right)^{2} \ldots\left(t_{k}-t_{k-1}\right)^{2} X_{\xi_{k-1}}^{(2 k-2)}, \frac{1}{(2 k-1) !}\left(t_{k}-t_{1}\right)^{2} \ldots\left(t_{k}-t_{k-1}\right)^{2} X_{\eta_{k-1}}^{(2 k-1)}\right)^{T}= \\
=\frac{\widetilde{\Delta}}{[2 ! \ldots(2 k-1) !]^{2}} \prod_{1 \leq i<j \leq k}\left(t_{j}-t_{i}\right)^{8}
\end{gathered}
$$

with

$$
\begin{aligned}
\widetilde{\Delta}=\operatorname{det} \operatorname{Var}\left(X_{t_{1}}, X_{t_{1}}^{\prime}, X_{\xi_{1}}^{(2)}, X_{\eta_{2}}^{(3)}, \ldots, X_{\xi_{k-1}}^{(2 k-2)},\right. & \left.X_{\eta_{k-1}}^{(2 k-1)}\right)^{T} \rightarrow \\
& \rightarrow \operatorname{det} \operatorname{Var}\left(X_{t^{*}}, X_{t^{*}}^{\prime}, \ldots, X_{t^{*}}^{(2 k-1)}\right)^{T}
\end{aligned}
$$

as $t_{1}, t_{2}, \ldots, t_{k} \rightarrow t^{*}$. This proves $(22)$. 
Proposition 3.3 Suppose that $X$ is a centered Gaussian process with $C^{2 k-1}$ paths and that for each pairwise distinct values of the parameter $t_{1}, t_{2}, \ldots, t_{k} \in I$ the joint distribution of $\left(X_{t_{h}}, X_{t_{h}}^{\prime}, \ldots, X_{t_{h}}^{(2 k-1)}, h=1,2, \ldots, k\right)$ is non-degenerate. Then, as $t_{1}, t_{2}, \ldots, t_{k} \rightarrow t^{*}$ :

$$
\widetilde{A}_{t_{1}, \ldots, t_{k}}^{+}(0, \ldots, 0) \approx J_{k}\left(t^{*}\right) \prod_{1 \leq i<j \leq k}\left(t_{j}-t_{i}\right)^{4}
$$

where $J_{k}(t)$ is a continuous non-zero function of $t$.

Proof. Introduce the notation

$$
D_{k}(t)=\operatorname{det} \operatorname{Var}\left(X_{t}, X_{t}^{\prime}, \ldots, X_{t}^{(k)}\right)^{T}
$$

In the same way as in the proof of Proposition 3.2 and with a simpler computation, it follows that as $t_{1}, t_{2}, \ldots, t_{k} \rightarrow t^{*}$

$$
\operatorname{det} \operatorname{Var}\left(X_{t_{1}}, X_{t_{2}}, \ldots, X_{t_{k}}\right)^{T} \approx \frac{1}{[2 ! \ldots . .(k-1) !]^{2}}\left[\prod_{1 \leq i<j \leq k}\left(t_{j}-t_{i}\right)^{2}\right] . D_{k-1}\left(t^{*}\right)
$$

For pairwise different values $t_{1}, t_{2}, \ldots, t_{k}$, let $Z=\left(Z_{1}, \ldots, Z_{k}\right)^{T}$ be a random vector having the conditional distribution of $\left(X_{t_{1}}^{\prime}, \ldots, X_{t_{k}}^{\prime}\right)^{T}$ given $X_{t_{1}}=X_{t_{2}}=\ldots=X_{t_{k}}=$ 0 . The (Gaussian) distribution of $Z$ is centered and we denote its covariance matrix by $\Sigma$. Also put:

$$
\Sigma^{-1}=\frac{1}{\operatorname{det}(\Sigma)}\left(\sigma^{i j}\right)_{i, j=1, \ldots, k}
$$

$\sigma^{i j}$ being the cofactor of the position $(i, j)$ in the matrix $\Sigma$. Then, one can write:

$$
\widetilde{A}_{t_{1}, \ldots, t_{k}}^{+}(0, \ldots, 0)=E\left\{Z_{1}^{+} \ldots Z_{k}^{+}\right\} \cdot p_{X_{t_{1}}, \ldots, X_{t_{k}}}(0, \ldots, 0)
$$

and

$$
\widetilde{A}_{t_{1}, \ldots, t_{k}}^{+}(0, \ldots, 0)=\frac{1}{(2 \pi)^{\frac{k}{2}}(\operatorname{det}(\Sigma))^{\frac{1}{2}}} \int_{\left(R^{+}\right)^{k}} x_{1} \ldots x_{k} \exp \left[-\frac{F\left(x_{1}, \ldots, x_{k}\right)}{2 . \operatorname{det}(\Sigma)}\right] d x_{1} \ldots d x_{k}
$$

where

$$
F\left(x_{1}, \ldots, x_{k}\right)=\sum_{i, j=1}^{k} \sigma^{i j} x_{i} x_{j}
$$


Letting $t_{1}, t_{2}, \ldots, t_{k} \rightarrow t^{*}$ and using (22) and (26) we get:

$$
\begin{aligned}
& \operatorname{det}(\Sigma)=\frac{\operatorname{det} \operatorname{Var}\left(X_{t_{1}}, X_{t_{1}}^{\prime}, \ldots, X_{t_{k}}, X_{t_{k}}^{\prime}\right)^{T}}{\operatorname{det} \operatorname{Var}\left(X_{t_{1}}, \ldots, X_{t_{k}}\right)^{T}} \\
& \approx \frac{1}{[k ! \ldots . .(2 k-1) !]^{2}}\left[\prod_{1 \leq i<j \leq k}\left(t_{j}-t_{i}\right)^{6}\right] \cdot \frac{D_{2 k-1}\left(t^{*}\right)}{D_{k-1}\left(t^{*}\right)}
\end{aligned}
$$

We consider now the behaviour of the $\sigma^{i j}(i, j=1, \ldots, k)$. Let us first look at $\sigma^{11}$. Using the same method as above, now applied to the cofactor of the position $(1,1)$ in $\Sigma$, one has:

$$
\begin{aligned}
& \sigma^{11}= \frac{\operatorname{det} \operatorname{Var}\left(X_{t_{1}}, X_{t_{2}}, \ldots, X_{t_{k}}, X_{t_{2}}^{\prime}, \ldots, X_{t_{k}}^{\prime}\right)^{T}}{\operatorname{det} \operatorname{Var}\left(X_{t_{1}}, \ldots, X_{t_{k}}\right)^{T}} \approx \\
& \approx \frac{\frac{1}{[2 ! \ldots(2 k-2) !]^{2}}\left[\prod_{2 \leq i<j \leq k}\left(t_{j}-t_{i}\right)^{8}\right]\left[\prod_{2 \leq h \leq k}\left(t_{1}-t_{h}\right)^{4}\right] D_{2 k-2}\left(t^{*}\right)}{\frac{1}{[2 ! \ldots . .(k-1) !]^{2}}\left[\prod_{1 \leq i<j \leq k}\left(t_{j}-t_{i}\right)^{2}\right] D_{k-1}\left(t^{*}\right)}= \\
&=\frac{1}{[k ! \ldots(2 k-2) !]^{2}}\left[\prod_{2 \leq i<j \leq k}\left(t_{j}-t_{i}\right)^{6}\right]\left[\prod_{2 \leq h \leq k}\left(t_{1}-t_{h}\right)^{2}\right] \frac{D_{2 k-2}\left(t^{*}\right)}{D_{k-1}\left(t^{*}\right)}
\end{aligned}
$$

A similar computation holds for $\sigma^{i i}, i=2, \ldots, k$.

Consider now $\sigma^{12}$. One has:

$$
\begin{aligned}
& \sigma^{12}=-\frac{\operatorname{det}\left[E\left\{\left(X_{t_{1}}, X_{t_{2}}, \ldots, X_{t_{k}}, X_{t_{2}}^{\prime}, \ldots, X_{t_{k}}^{\prime}\right)^{T} \cdot\left(X_{t_{1}}, X_{t_{2}}, \ldots, X_{t_{k}}, X_{t_{1}}^{\prime}, X_{t_{3}}^{\prime} \ldots, X_{t_{k}}^{\prime}\right)\right\}\right]}{\operatorname{det} \operatorname{Var}\left(X_{t_{1}}, \ldots, X_{t_{k}}\right)^{T}}= \\
& =\frac{\operatorname{det}\left[E\left\{\left(X_{t_{2}}, X_{t_{2}}^{\prime}, \ldots, X_{t_{k}}, X_{t_{k}}^{\prime}, X_{t_{1}}\right)^{T} \cdot\left(X_{t_{1}}, X_{t_{1}}^{\prime}, X_{t_{3}}, X_{t_{3}}^{\prime}, \ldots, X_{t_{k}}, X_{t_{k}}^{\prime}, X_{t_{2}}\right)\right\}\right]}{\operatorname{det} \operatorname{Var}\left(X_{t_{1}}, \ldots, X_{t_{k}}\right)^{T}} \approx \\
& \approx \frac{1}{[k ! \ldots(2 k-2) !]^{2}}\left[\prod_{3 \leq i<j \leq k}\left(t_{j}-t_{i}\right)^{6}\right]\left[\prod_{3 \leq h \leq k}\left(t_{1}-t_{h}\right)^{4}\left(t_{2}-t_{h}\right)^{4}\right]\left(t_{2}-t_{1}\right)^{2} \cdot \frac{D_{2 k-2}\left(t^{*}\right)}{D_{k-1}\left(t^{*}\right)}
\end{aligned}
$$

A similar computation applies to all the cofactors $\sigma^{i j}, i \neq j$.

Perform in the integral in (28) the change of variables

$$
x_{j}=\left[\prod_{i=1, i \neq j}^{i=k}\left(t_{i}-t_{j}\right)^{2}\right] \cdot y_{j} \quad j=1, \ldots, k
$$


and the integral becomes:

$$
\left[\prod_{1 \leq i<j \leq k}\left(t_{j}-t_{i}\right)^{8}\right] \int_{\left(R^{+}\right)^{k}} y_{1} \ldots y_{k} \exp \left[-\frac{1}{2 \cdot \operatorname{det}(\Sigma)} G\left(y_{1}, \ldots, y_{k}\right)\right] d y_{1} \ldots d y_{k}
$$

where

$$
G\left(y_{1}, \ldots, y_{k}\right)=\sum_{i, j=1}^{k} \sigma^{i j}\left[\prod_{h=1, h \neq i}^{h=k}\left(t_{h}-t_{i}\right)^{2}\right]\left[\prod_{h=1, h \neq j}^{h=k}\left(t_{h}-t_{j}\right)^{2}\right] y_{i} y_{j}
$$

so that, as $t_{1}, t_{2}, \ldots, t_{k} \rightarrow t^{*}$

$$
\frac{G\left(y_{1}, \ldots, y_{k}\right)}{\operatorname{det}(\Sigma)} \approx[(2 k-1) !]^{2} \frac{D_{2 k-2}\left(t^{*}\right)}{D_{2 k-1}\left(t^{*}\right)}\left(\sum_{i=1}^{i=k} y_{i}\right)^{2}
$$

Now, passage to the limit under the integral sign in (28), which is easily justified by application of the Lebesgue Theorem, leads to

$$
E\left\{Z_{1}^{+} \ldots Z_{k}^{+}\right\} \approx \frac{1}{(2 \pi)^{\frac{k}{2}}} k ! \ldots(2 k-1) !\left[\prod_{1 \leq i<j \leq k}\left|t_{j}-t_{i}\right|^{5}\right]\left(\frac{D_{k-1}\left(t^{*}\right)}{D_{2 k-1}\left(t^{*}\right)}\right)^{\frac{1}{2}} I_{k}\left(\alpha^{*}\right)
$$

where $I_{k}(\alpha), \alpha>0$ is

$$
I_{k}(\alpha)=\int_{\left(R^{+}\right)^{k}} y_{1} \ldots y_{k} \exp \left[-\frac{\alpha}{2}\left(\sum_{i=1}^{i=k} y_{i}\right)^{2}\right] d y_{1} \ldots d y_{k}=\frac{1}{\alpha^{k}} I_{k}(1)
$$

and

$$
\alpha^{*}=[(2 k-1) !]^{2} \frac{D_{2 k-2}\left(t^{*}\right)}{D_{2 k-1}\left(t^{*}\right)}
$$

Replacing into (27) one gets the result with

$$
J_{k}(t)=\frac{2 ! \ldots(2 k-2) !}{[2 \pi(2 k-1) !]^{2 k-1}} \frac{I_{k}(1)}{\left[D_{2 k-1}(t)\right]^{\frac{1}{2}}}\left[\frac{D_{2 k-1}(t)}{D_{2 k-2}(t)}\right]^{k}
$$

This finishes the proof. 


\section{Numerical examples}

\subsection{Comparison with Monte-Carlo method}

First, let us compare the numerical computation based upon Theorem 2.1 with the Monte-Carlo method based on the simulation of the paths. We do this for stationary Gaussian processes that satisfy the hypotheses of Theorem 2.2 and also the non-degeneracy condition that ensures that one is able to compute the factorial moments of crossings by means of Rice formulae.

Suppose that we want to compute $P(M>u)$ with an error bounded by $\delta$, where $\delta>0$ is a given positive number.

To proceed by simulation, we discretize the paths by means of a uniform partition $\left\{t_{j}:=j / n, j=0,1, \ldots, n\right\}$. Denote

$$
M^{(n)}:=\sup _{0 \leq j \leq n} X_{t_{j}} .
$$

Using Taylor's formula at the time where the maximum $M$ of $X_{(.)}$occurs, one gets :

$$
0 \leq M-M^{(n)} \leq\left\|X^{\prime \prime}\right\|_{\infty} /\left(2 n^{2}\right)
$$

It follows that

$$
\begin{aligned}
0 \leq P(M>u)-P\left(M^{(n)}>u\right)=P(M>u, & \left.M^{(n)} \leq u\right) \leq \\
& \leq P\left(u<M \leq u+\left\|X^{\prime \prime}\right\|_{\infty} /\left(2 n^{2}\right)\right) .
\end{aligned}
$$

If we admit that the distribution of $M$ has a locally bounded density (which is a well-known fact under the mentioned hypotheses) the above suggests that a number of $n=$ (const) $\delta^{-1 / 2}$ points is required if one wants the mean error $P(M>u)-$ $P\left(M^{(n)}>u\right)$ to be bounded by $\delta$.

On the other hand, to estimate $P\left(M^{(n)}>u\right)$ by Monte-Carlo with a mean square error smaller than $\delta$, we require the simulation of $N=$ (const) $\delta^{-2}$ Gaussian n-tuples $\left(X_{t_{1}}, \ldots, X_{t_{n}}\right)$ from the distribution determined by the given stationary process. Performing each simulation demands (const)nlog $(n)$ elementary operations (Dietrich and Newsam, 1997). Summing up, the total mean number of elementary operations required to get a mean square error bounded by $\delta$ in the estimation of $P(M>u)$ has the form (const) $\delta^{-5 / 2} \log (1 / \delta)$.

Suppose now that we apply Theorem 2.1 to a Gaussian stationary centered process verifying the hypotheses of Theorem 2.2 and the non-degeneracy condition. 
The bound for $\gamma_{m}^{*}$ in Equation (10) implies that computing a partial sum with (const) $\log (1 / \delta)$ terms assures that the tail in the Rice series is bounded by $\delta$. If one computes each $\nu_{m}$ by means of a Monte-Carlo method for the multiple integrals appearing in the Rice formulae, then the number of elementary operations for the whole procedure will have the form (const) $\delta^{-2} \log (1 / \delta)$. Hence, this is better than simulation as $\delta$ tends to zero.

As usual, for given $\delta>0$, the value of the generic constants decides the comparison between both methods.

More important is the fact that the enveloping property of the Rice series implies that the actual number of terms required by the application of Theorem 2.1 can be much smaller than the one resulting from the a priori bound on $\gamma_{m}^{*}$. More precisely, suppose that we have obtained each numerical approximation $\nu_{m}^{*}$ of $\nu_{m}$ with a precision $\eta$

$$
\left|\nu_{m}^{*}-\nu_{m}\right| \leq \eta
$$

and that we stop when

$$
\frac{\nu_{m_{0}+1}^{*}}{\left(m_{0}+1\right) !} \leq \eta
$$

Then, it follows that

$$
\left|\sum_{m=1}^{\infty}(-1)^{m+1} \frac{\nu_{m}}{m !}-\sum_{m=1}^{m_{0}}(-1)^{m+1} \frac{\nu_{m}^{*}}{m !}\right| \leq(e+1) \eta .
$$

Putting $\eta=\delta /(e+1)$, we get the desired bound. In other words one can profit of the successive numerical approximations of $\nu_{m}$ to determine a new $m_{0}$ which turns out to be - in certain interesting examples - much smaller than the one deduced from the a priori bound on $\gamma_{m}^{*}$.

\subsection{Comparison with usuals bounds}

Next, we will give the results of the evaluation of $P\left(M_{T}>u\right)$ using up to three terms in the Rice series in a certain number of typical cases. We compare these results with the classical evaluation using what is often called the "Davies (1977) bound". In fact this bound seems to have been widely used since the work of Rice (1944). It is an upper-bound with no control on the error, given by:

$$
P(M>u) \leq P\left(X_{0}>u\right)+E\left(U_{u}([0, T])\right)
$$


The above mentioned result by Piterbarg (11) shows that in fact, for fixed $T$ and high level $u$ this bound is sharp. In general, using more than one term of the Rice series supplies a remarkable improvement in the computation.

We consider several stationary centered Gaussian processes listed in the following table, where the covariances and the corresponding spectral densities are indicated.

\begin{tabular}{lll} 
process & covariance & spectral density \\
\hline$X_{1}$ & $\Gamma_{1}(t)=\exp \left(-t^{2} / 2\right)$ & $f_{1}(x)=(2 \pi)^{-1 / 2} \exp \left(-x^{2} / 2\right)$ \\
$X_{2}$ & $\Gamma_{2}(t)=(\operatorname{ch}(t))^{-1}$ & $f_{2}(x)=(2 \operatorname{ch}((\pi x) / 2))^{-1}$ \\
$X_{3}$ & $\Gamma_{3}(t)=\left(3^{1 / 2} t\right)^{-1} \sin \left(3^{1 / 2} t\right)$ & $f_{3}(x)=12^{-1 / 2} \mathbb{I}_{\{-\sqrt{3}<x<\sqrt{3}\}}$ \\
$X_{4}$ & $\Gamma_{4}(t)=e^{-|\sqrt{5} t|}\left(\frac{\sqrt{5}}{3}|t|^{3}+2 t^{2}+\sqrt{5}|t|+1\right)$ & $f_{4}(x)=\frac{10^{4}}{\sqrt{5} \pi}\left(5+x^{2}\right)^{-4}$
\end{tabular}

In all cases, $\lambda_{0}=\lambda_{2}=1$ to be able to compare the various results. Note that $\Gamma_{1}$ and $\Gamma_{3}$ have analytic extensions to the whole plane, so that Theorem 2.2 applies to the processes $X_{1}$ and $X_{3}$. On the other hand, even though all spectral moments of the process $X_{2}$ are finite, Theorem 2.2 applies only for a length less than $\pi / 4$ since the meromorphic extension of $\Gamma_{2}($.$) has poles at the points i \pi / 2+k \pi i, k$ an integer. With respect to $\Gamma_{4}($.$) notice that it is obtained as the convolution \Gamma_{5} * \Gamma_{5} * \Gamma_{5} * \Gamma_{5}$ where $\Gamma_{5}(t):=e^{-|t|}$ is the covariance of the Ornstein-Uhlenbeck process, plus a change of scale to get $\lambda_{0}=\lambda_{2}=1$. The process $X_{4}$ has $\lambda_{6}<\infty$ and $\lambda_{8}=\infty$ and its paths are $C^{3}$. So, for the processes $X_{2}$ and $X_{4}$ we apply Theorem 2.3 to compute $F(T, u)$.

Table 1 contains the results for $T=1,4,6,8,10$ and the values $u=-2,-1,0,1,2,3$ using three terms of the Rices series. A single value is given when a precision of $10^{-2}$ is met; otherwise the lower-bound and the upper-bound given by two or three terms of the Rices series respectively, are diplayed. The calculation uses a deterministic evaluation of the first two moments $\nu_{1}$ and $\nu_{2}$ using program written by Cierco Croquette and Delmas (2000) and a Monte-Carlo evaluation of $\nu_{3}$. In fact, for simpler and faster calculation, $\tilde{\nu}_{3}$ has been evaluated instead of $\nu_{3}$ providing a slightly weaker bound.

In addition Figures 1 to 4 show the behavior of four bounds : namely, from the highest to the lowest

- The Davies bound $(D)$ defined by formula (30) 


\begin{tabular}{c||l|l|l|l|l|}
\multicolumn{1}{c||}{} & \multicolumn{5}{c}{ Length of the time interval } \\
\hline $\mathrm{u}$ & 1 & 4 & 6 & 8 & 10 \\
\hline \hline-2 & 0.99 & 1.00 & 1.00 & 1.00 & 1.00 \\
& 0.99 & 1.00 & 1.00 & 1.00 & 1.00 \\
& 1.00 & 1.00 & 1.00 & 1.00 & 1.00 \\
& 0.99 & 1.00 & 1.00 & 1.00 & 1.00 \\
\hline-1 & 0.93 & 1.00 & 1.00 & $0.99-1.00$ & $0.98-1.00$ \\
& 0.93 & 0.99 & 1.00 & $0.99-1.00$ & $0.98-1.00$ \\
& 0.93 & 1.00 & 1.00 & 1.00 & 0.99 \\
& 0.93 & 1.00 & 1.00 & $0.99-1.00$ & $0.98-1.00$ \\
\hline 0 & 0.65 & 0.90 & 0.95 & $0.95-0.99$ & $0.90-1.00$ \\
& 0.65 & 0.89 & $0.94-0.95$ & $0.93-0.99$ & $0.87-1.00$ \\
& 0.656 & 0.919 & 0.97 & $0.98-0.99$ & $0.92-1.00$ \\
& 0.65 & 0.89 & $0.94-0.95$ & $0.94-0.99$ & $0.88-1.00$ \\
\hline 1 & 0.25 & 0.49 & 0.61 & $0.69-0.70$ & $0.74-0.77$ \\
& 0.25 & 0.48 & 0.58 & $0.66-0.68$ & $0.70-0.76$ \\
& 0.26 & 0.51 & 0.62 & 0.71 & $0.76-0.78$ \\
& 0.25 & 0.48 & 0.59 & $0.67-0.69$ & $0.72-0.77$ \\
\hline 2 & 0.04 & 0.11 & 0.15 & 0.18 & 0.22 \\
& 0.04 & 0.11 & 0.14 & 0.18 & 0.21 \\
& 0.04 & 0.11 & 0.15 & 0.19 & 0.22 \\
& 0.04 & 0.11 & 0.14 & 0.18 & 0.22 \\
\hline \multirow{2}{*}{3} & 0.00 & 0.01 & 0.01 & 0.02 & 0.02 \\
& 0.00 & 0.01 & 0.01 & 0.02 & 0.02 \\
& 0.00 & 0.01 & 0.01 & 0.02 & 0.02 \\
& 0.00 & 0.01 & 0.01 & 0.02 & 0.02 \\
\hline
\end{tabular}

Table 1: Values of $P(M>u)$ for the different processes. Each cell contains, from top to bottom, the values corresponding to stationary centered Gaussian processes with covariances $\Gamma_{1}, \Gamma_{2}, \Gamma_{3}$ and $\Gamma_{4}$ respectively. The calculation uses three terms of the Rice series for the upper-bound and two terms for the lower-bound. Both bounds are rounded up to two decimals and when they differ, both displayed. 
- One, three, or two terms of the Rice series $(R 1, R 3, R 2$ in the sequel) that is

$$
P\left(X_{0}>u\right)+\sum_{m=1}^{K}(-1)^{m+1} \frac{\nu_{m}}{m !}
$$

with $K=1,3$ or 2 .

Note that the bound $D$ differs from $R 1$ due to the difference between $\nu_{1}$ and $\tilde{\nu}_{1}$. These bounds are evaluated for $T=4,6,8,10,15$ and also for $T=20$ and $T=40$ when they fall in the range $[0,1]$. Between these values an ordinary spline interpolation has been performed.

In addition we illustrate the complete detailed calculation in three chosen cases. They correspond to zero and positive levels $u$. For $u$ negative, it is easy to check that the Davies bound is often greater than 1, thus non informative.

- For $u=0, T=6, \Gamma=\Gamma_{1}$, we have $P\left(X_{0}>u\right)=0.5, \tilde{\nu}_{1}=0.955, \nu_{1}=0.602$, $\nu_{2} / 2=.150, \tilde{\nu}_{3} / 6=0.004$, so that:

$$
D=1.455, R 1=1.103, R 3=0.957, R 2=0.953
$$

$R 2$ and $R 3$ give a rather good evaluation of the probability, the Davies bound gives no information.

- For $u=1.5, T=15, \Gamma=\Gamma_{2}$, we have $P\left(X_{0}>u\right)=0.067, \tilde{\nu}_{1}=0.517$, $\nu_{1}=0.488, \nu_{2} / 2=0.08, \tilde{\nu}_{3} / 6=0.013$, so that:

$$
D=0.584, R 1=0.555, R 3=0.488, R 2=0.475
$$

In this case the Davies bound is not sharp and a very clear improvement is provided by the two bounds $R 2$ and $R 3$.

- For $u=2, T=10, \Gamma=\Gamma_{3}$, we have $P\left(X_{0}>u\right)=0.023, \tilde{\nu}_{1}=0.215$, $\nu_{1}=0.211, \nu_{2} / 2=0.014, \tilde{\nu}_{3} / 6=310^{-4}$, so that:

$$
D=0.238, R 1=0.234, R 3=0.220 ., R 2=0.220
$$

In this case the Davies bound is rather sharp.

As a conclusion, these numerical results show that it is worth using several terms of the Rice series. In particular the first three terms are relatively easy to compute and provide a good evaluation of the distribution of $M$ under a rather broad set of conditions. 


\section{Acknowledgements}

We thank C. Delmas for computational assistance. This work has received a support ECOS program U97E02.

\section{References}

Adler, R.J. (1990). An Introduction to Continuity, Extrema and Related Topics for General Gaussian Processes, IMS, Hayward, Ca.

Adler, R.J. (2000) On excursion sets, tube formulae, and maxima of random fields. Annals of Applied Probability. To appear

Azaïs, J-M., Cierco, C. and Croquette, A. (1999). Bounds and asymptotic expansions for the distribution of the maximum of a smooth stationary Gaussian process. ESAIM: Probability and Statistics, 3, 107-129.

Azaïs, J-M. and Wschebor M. (1997). Une formule pour calculer la distribution du maximum d'un processus stochastique. C.R. Acad. Sci. Paris, t. 324, sérieI, $225-230$.

Azaïs, J-M. and Wschebor M. (1999). Régularité de la loi du maximum de processus gaussiens réguliers. C.R. Acad. Sci. Paris, t. 328, Sér. I, 333-336.

Azaïs, J-M and Wschebor, M. (2000). On the Regularity of the Distribution of the Maximum of one-parameter Gaussian Processes, accepted for publication at Probability Theory and Related Fields.

Azaïs, J-M, Bardet, J-M and Wschebor, M. (2000). On the Tails of the Distribution of the Maximum of a Smooth Stationary Gaussian Process, submitted.

Belyaev, Yu. (1966). On the number of intersections of a level by a Gaussian Stochastic process. Theory Prob. Appl., 11, 106-113.

Berman, S.M. (1985a). An asymptotic formula for the distribution of the maximum of a Gaussian process with stationary increments. J. Appl. Prob., 22,454-460.

Berman, S.M. (1985b). The maximum of a Gaussian process with nonconstant variance. Ann. Inst. H. Poincaré Probab. Statist., 21, 383-391.

Berman, S.M. (1992). Sojourns and extremes of stochastic processes, The Wadworth and Brooks, Probability Series.

Berman, S.M. and Kôno, N. (1989). The maximum of a gaussian process with non-constant variance: a sharp bound for the distribution of the tail. Ann. Probab., $17,632-650$.

Borell, C. (1975). The Brunn-Minkowski inequality in Gauss space. Invent. Math., 30, 207-216. 
Cramér, H. and Leadbetter, M.R. (1967). Stationary and Related Stochastic Processes, J. Wiley \& Sons, New-York.

Cierco, C. (1996). Problèmes statistiques liés à la détection et à la localisation d'un gène à effet quantitatif. PHD dissertation. University of Toulouse, France.

Cierco-Ayrolles, C., Croquette, A. and Delmas, C. (2000). Computing the Distribution of the Maximum of Regular Gaussian Processes. Submitted.

Cressie, N (1980). The asymptotic distribution of the scan statistic under uniformity. Ann. Probab, 8, 828-840.

Cuzick, J. (1975). Conditions for finite moments of the number of zero crossings for Gaussian processes.Ann. Probab, 3, 849-858.

Darling, D. A.(1983). On the supremum of certain Gaussian processes. Ann. Probab. 11, 803-806.

Davies, R.B. (1987). Hypothesis testing when a nuisance parameter is present only under the alternative. Biometrika 74, 33-43.

Davis, J. D. (1975). Interpolation and approximation. Dover, New York.

DeLong, D. M. (1981). Crossing probabilities for a square root boundary by a Bessel Process. Communication in Statistics-Theory and methods. A10, 2197-2213.

Diebolt, J. and Posse, C. (1996). On the Density of the Maximum of Smooth Gaussian Processes.Ann. Probab., 24, 1104-1129.

Dietrich, C. R. and Newsam G. N. (1997.) Fast and exact simulation of stationary Gaussian processes throught circulant embedding of the covartiance matrix. . SIAM J. Sci. Comput. 18, 4, 1088-1107.

Fernique, X.(1974). Régularité des trajectoires des fonctions aléatoires gaussiennes.Ecole d'Eté de Probabilités de Saint Flour. Lecture Notes in Mathematics, 480, Springer-Verlag,New-York.

Goldman, M. (1971). On the first passage of the integrated Wiener process. The Annals Math. Statist., 42, 6, 2150-2155.

Lachal, A. (1991). Sur le premier instant de passage de l'intégrale du mouvement Brownien. Ann. Inst. H. Poincaré Probab. Statist. 27, 385-405.

Landau, H.J. and Shepp, L.A (1970). On the supremum of a Gaussian process. Sankya Ser. A 32, 369-378.

Leadbetter, M.R., Lindgren, G. and Rootzén, H. (1983). Extremes and related properties of random sequences and processes. Springer-Verlag, New-York.

Ledoux, M. (1996). Isoperimetry and Gaussian Analysis. Ecole d'Eté de Probabilités de Saint-Flour 1994. Lecture Notes in Math. 1648, 165-264. Springer-Verlag. New York.

Ledoux, M. and Talagrand, M. (1991). Probability in Banach Spaces, SpringerVerlag, New-York. 
Lifshits, M.A.(1995). Gaussian random functions . Kluwer, The Netherlands.

Lindgren, G. (1972). Wave-length and Amplitude in Gaussian Noise . Adv. Appl. Prob., 4, 81-108.

McKean, H.P. (1963). A winding problem for a resonant driven by a white noise. J. Math. Kyoto Univ., 2, 227-235.

Marcus, M.B. (1977). Level Crossings of a Stochastic Process with Absolutely Continuous Sample Paths, Ann. Probab., 5, 52-71.

Marcus, M.B. and Shepp, L.A. (1972). Sample behaviour of Gaussian processes. Proc. Sith Berkeley Symp. Math. Statist. Prob., 2, 423-442.

Miroshin, R. N. (1974). Rice series in the theory of random functions. Vestnik Leningrad Univ. Math., 1, 143-155.

Miroshin, R. N. (1977). Condition for finiteness of moments of the number of zeros of stationary Gaussian processes. Th. Prob. Appl., 22 , 615-624.

Miroshin, R. N. (1983). The use of Rice series. Th. Prob. Appl. 28, 714-726.

Nualart, D. and Wschebor, M. (1991). Intégration par parties dans l'espace de Wiener et approximation du temps local. Prob. Th. Rel. Fields, 90, 83-109.

Piterbarg, V; I. (1981). Comparison of distribution functions of maxima of Gaussian processes. Th, Proba. Appl., 26, 687-705.

Piterbarg, V; I. (1996). Asymptotic Methods in the Theory of Gaussian Processes and Fields. American Mathematical Society. Providence, Rhode Island.

Qualls, C and Watanabe, H. (1973). Asymptotic properties of Gaussian process. Ann. Math. Statistics, 43, 580-596

Rice, S.O. (1944-1945). Mathematical Analysis of Random Noise.Bell System Tech. J., 23, 282-332; 24, 45-156.

Shepp, L. A. (1971). First passage time for a particular Gaussian process. The Ann. of Math. Stat., 42, 946-951.

Shepp, L. A. (1979). The joint density of the maximum and its location for a Wiener process with drift. J. Appl. Prob. 16, 423-427.

Shepp, L. A. and Slepian, D.(1976). First-passage time for a particular stationary periodic Gaussian process. J. Appl. Prob., 13, 27-38.

Slepian, D. (1961). First passage time for a particular Gaussian process.Ann. Math. Statist., 32, 610-612.

Slepian, D. (1962). The one-sided barrier problem for Gaussian noise.Bell System Tech. J. 42, 463-501.

Sun, J. (1993). Tail Probabilities of the Maxima of Gaussian Random Fields, Ann. Probab., 21, 34-71.

Talagrand, M. (1988). Small tails for the supremum of a Gaussian process. Ann. Inst. H. Poincaré, Sér. B, 24, 2, 307-315. 
Talagrand, M. (1996). Majorising measures: the general chaining. Ann. Probab. 24, 1049-1103.

Tsirelson, V.S. (1975). The Density of the Maximum of a Gaussian Process. Th. Probab. Appl., 20, 817-856.

Weber, M. (1985). Sur la densité du maximum d'un processus gaussien. J. Math. Kyoto Univ., 25, 515-521.

Wschebor, M. (1985). Surfaces aléatoires. Mesure géometrique des ensembles de niveau.Lecture Notes in Mathematics, 1147, Springer-Verlag.

Wschebor, M. (2000). Sur la loi du sup de certains processus Gaussiens non bornés. Accepted for publication by C.R. Acad. Sci. Paris.

Ylvisaker, D. (1968). A Note on the Absence of Tangencies in Gaussian Sample Paths. The Ann. of Math. Stat., 39, 261-262. 


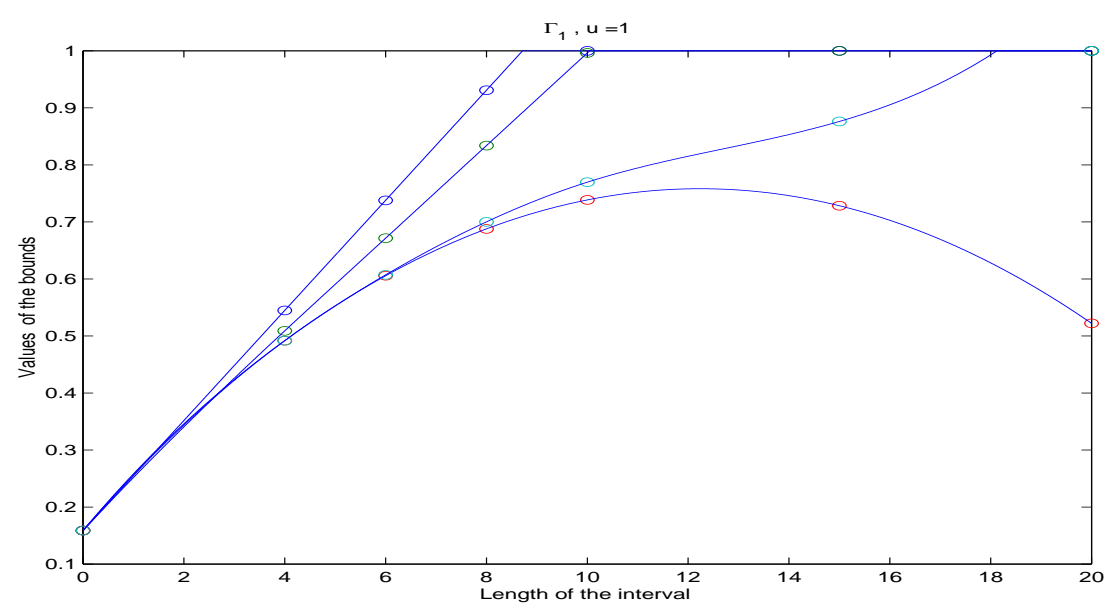

Figure 1: For the process with covariance $\Gamma_{1}$ and the level $u=1$, representation of the three upper-bounds D, R1, R3 and the lower-bound R2 (from top to bottom) as a function of the length $T$ of the interval

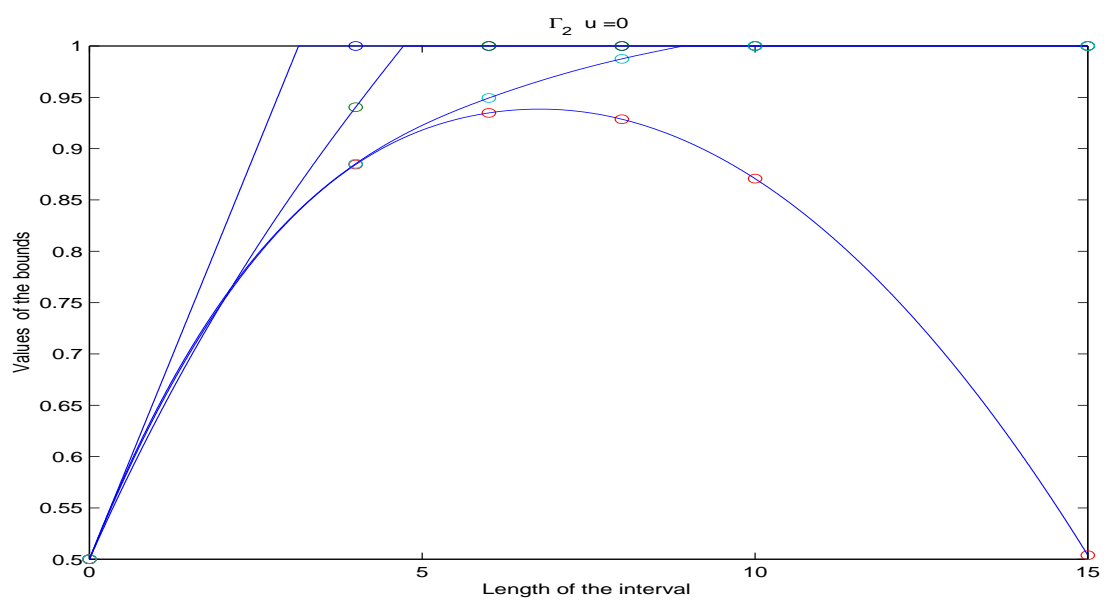

Figure 2: For the process with covariance $\Gamma_{2}$ and the level $u=0$, representation of the three upper-bounds: D, R1, R3 and the lower-bound R2 (from top to bottom) as a function of the length $T$ of the interval 


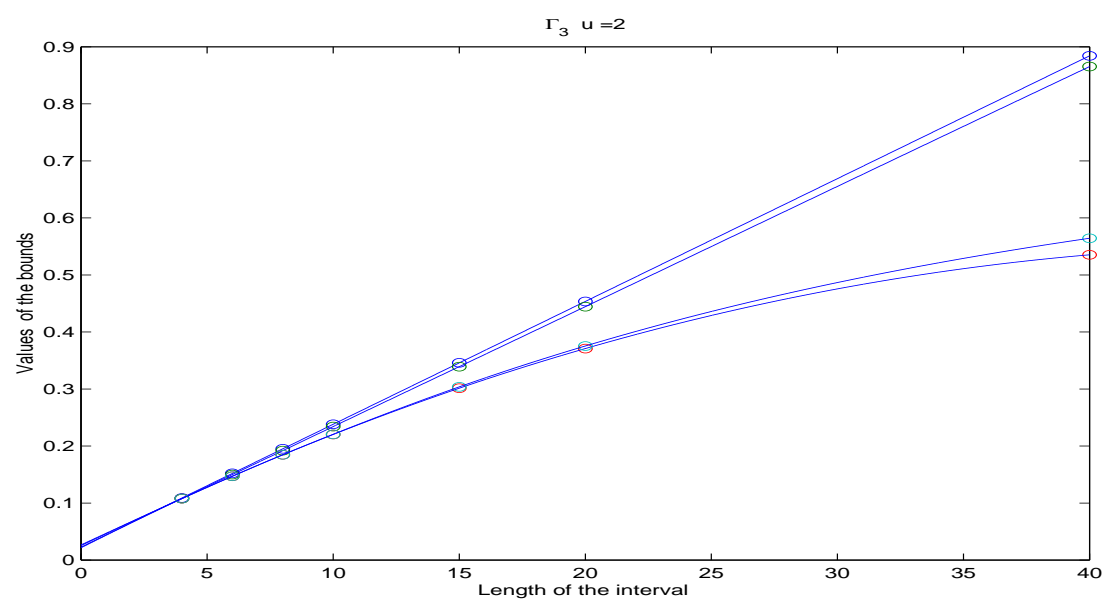

Figure 3: For the process with covariance $\Gamma_{3}$ and the level $u=2$, representation of the three upper-bounds: D, R1, R3 and the lower-bound R2 (from top to bottom) as a function of the length $T$ of the interval

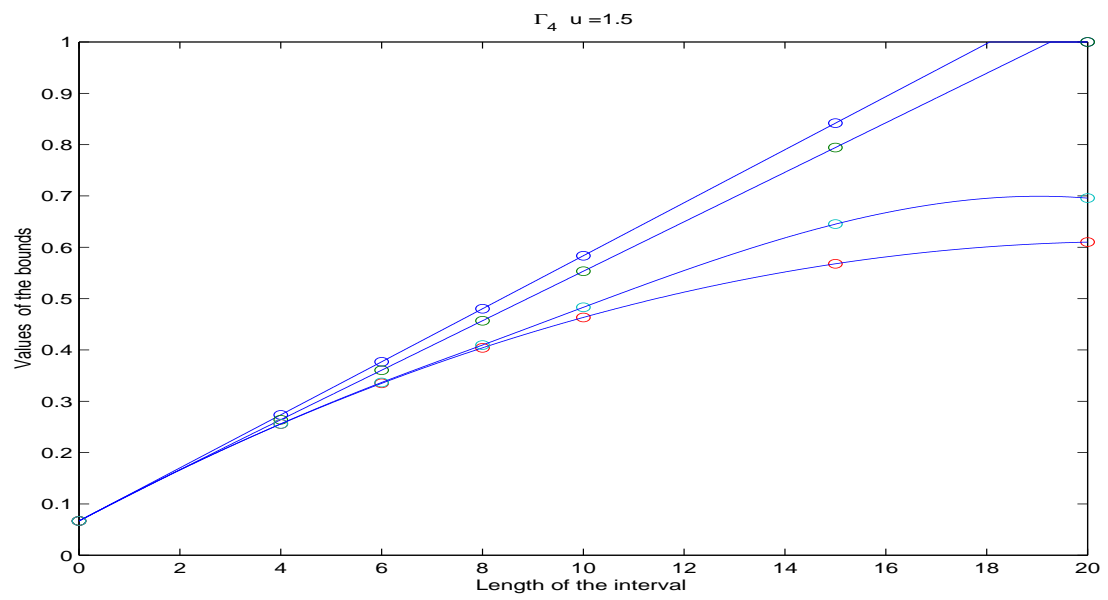

Figure 4: For the process with covariance $\Gamma_{4}$ and the level $u=1.5$, representation of the three upper-bounds: D, R1, R3 and the lower-bound R2 (from top to bottom) as a function of the length $T$ of the interval 